\title{
SOME REMARKS ON GENERALIZED METRIC SPACES OF BRANCIARI
}

\author{
POOM KUMAM AND NGUYEN VAN DUNG
}

\begin{abstract}
In this paper, we prove some properties of a generalized metric space in the sense of Branciari in [1]. As applications, we correct some confusion about this space in the literature. Examples are given to illustrate the results.
\end{abstract}

\section{INTRODUCTION AND PRELIMINARIES}

In 2000, Branciari [1] introduced the following notion of an $n$-generalized metric space.

Definition 1.1 ([1], Definition 2.1). Let $X$ be a non-empty set and $d$ : $X \times X \longrightarrow[0,+\infty)$ be a map that satisfy the following

(1) $d(x, y)=0$ if and only if $x=y$ for all $x, y \in X$.

(2) $d(x, y)=d(y, x)$ for all $x, y \in X$.

(3) $d(x, y) \leq d\left(x, u_{1}\right)+d\left(u_{1}, u_{2}\right)+\ldots+d\left(u_{n}, y\right)$ for all $x, y \in X$ and all distinct points $u_{1}, \ldots, u_{n} \in X$ each of them different from $x$ and $y$.

Then $d$ is called an n-generalized metric on $X$ and $(X, d)$ is called an $n$ generalized metric space, or for short, n-g.m.s. A sequence $\left\{x_{n}\right\}$ is called convergent to $x$ in $(X, d)$ if $\lim _{n \rightarrow \infty} d\left(x_{n}, x\right)=0$. A sequence $\left\{x_{n}\right\}$ is called Cauchy if $\lim _{n, m \rightarrow \infty} d\left(x_{n}, x_{m}\right)=0$. The $n$-generalized metric space $(X, d)$ is called complete if every Cauchy sequence is a convergent sequence.

If $n=2$, an $n$-generalized metric space is called a generalized metric space, or for short, g.m.s, see [1, Definition 1.1].

The notion of the generalized metric space in the sense of Branciari was investigated by some authors and many fixed point theorems in such space were stated, see [9], [10] and references therein. In [1], Branciari claimed the following result without proof.

2010 Mathematics Subject Classification. Primary 46A16, 54H25; Secondary 54D99, $54 \mathrm{E} 99$.

Key words and phrases. Generalized metric space, fixed point. 
Proposition $1.2([1])$.

(1) A generalized metric space is a topological space with neighborhood basis given by $\mathcal{C}=\{B(x, r): x \in X, r>0\}$ where $B(x, r)=\{y \in$ $X: d(x, y)<r\}$ is the ball of center $x$ and radius $r$.

(2) A generalized metric d is continuous in each of its variables.

(3) A generalized metric space is a Hausdorff space.

In [15], an example was given to show that Proposition 1.2 is not true in general, also see [9, Example 1.2].

Example 1.3 ([15], Example 1.1). Let $A=\{0,2\}, B=\left\{\frac{1}{n}: n \in \mathbb{N}\right\}$ and $X=A \cup B$. Define $d: X \times X \longrightarrow \mathbb{R}$ as follows:

$$
d(x, y)= \begin{cases}0 & \text { if } x=y \\ 1 & \text { if } x \neq y \text { and }\{x, y\} \subset A \text { or }\{x, y\} \subset B \\ =d(y, x)=y & \text { if } x \in A, y \in B .\end{cases}
$$

Then we have

(1) $(X, d)$ is a complete, generalized metric space.

(2) $\lim _{n \rightarrow \infty} \frac{1}{n}=0$ and $\lim _{n \rightarrow \infty} \frac{1}{n}=2$.

(3) The sequence $\left\{\frac{1}{n}\right\}$ is not a Cauchy sequence.

(4) There does not exist $r>0$ such that $B(0, r) \cap B(2, r)=\emptyset$.

(5) $0 \in B\left(\frac{1}{3}, \frac{2}{3}\right)$ but there does not exist $r>0$ such that $B(0, r) \subset$ $B\left(\frac{1}{3}, \frac{2}{3}\right)$.

(6) $d$ is not continuous.

Note that, for a given generalized metric space $(X, d)$, we have the topology $\mathcal{T}_{d}$ induced by the convergence on $(X, d)$, that is, $\left(X, \mathcal{T}_{d}\right)$ is a sequential space in the sense of Franklin [8].

Definition $1.4([8])$. Let $X$ be a topological space.

(1) A subset $U$ of $X$ is called sequentially open if each sequence $\left\{x_{n}\right\}$ in $X$ converging to a point $x$ in $U$ is eventually in $U$, that is, there exists $n_{0}$ such that $x_{n} \in U$ for all $n \geq n_{0}$.

(2) A subset $F$ of $X$ is called sequentially closed if no sequence in $F$ converges to a point not in $F$.

(3) $X$ is called a sequential space if each sequentially open subset of $X$ is open, equivalently, each sequentially closed subset of $X$ is closed.

In a natural way, a generalized metric space $(X, d)$ is always understood to be the topological space $\left(X, \mathcal{T}_{d}\right)$.

One usual method of generating a topology is to use a neighborhood system as follows:

Proposition 1.5 ([6], Proposition 1.2.1). Suppose we are given a set $X$ and a family $\mathcal{B}$ of subsets of $X$ which has the properties: 
(B1) For any $U_{1}, U_{2} \in \mathcal{B}$ and every $x \in U_{1} \cap U_{2}$, there exists $U \in \mathcal{B}$ such that $x \in U \subset U_{1} \cap U_{2}$.

(B2) For every $x \in X$, there exists $U \in \mathcal{B}$ such that $x \in U$.

Let $\mathcal{O}$ be the family of all subsets of $X$ that are unions of subfamilies of $\mathcal{B}$. The family $\mathcal{O}$ is a topology on $X$ and the family $\mathcal{B}$ is a base for the topological space $(X, \mathcal{O})$.

We see that the family $\mathcal{B}$ of all finite intersections of the family $\mathcal{C}$ in Proposition 1.2 satisfies conditions (B1)-(B2). Then, by using Proposition 1.5, $\mathcal{B}$ is a basis of certain topology $\mathcal{T}^{d}$ on $X$. Recall that, for a metric space, $\mathcal{T}_{d}$ and $\mathcal{T}^{d}$ are coincident. But for a generalized metric space, $\mathcal{T}_{d}$ and $\mathcal{T}^{d}$ may not be coincident. In Example 1.3 and [9, Example 1.2], the authors used neighborhoods in $\left(X, \mathcal{T}^{d}\right)$ to prove the non-Hausdorff property of $\left(X, \mathcal{T}_{d}\right)$. Obviously this is a confused state of affairs.

In this paper, we prove some properties of a generalized metric space $(X, d)$ dependent on certain topologies on the set $X$. As applications, we correct the mentioned confusion about generalized metric spaces. Examples are given to illustrate the results.

\section{MAin RESUlts}

The relationship between $\mathcal{T}_{d}$ and $\mathcal{T}^{d}$ is as follows. Note that every ball $B(x, r), r>0$, is an open subset of $\left(X, \mathcal{T}^{d}\right)$.

Proposition 2.1. Let $(X, d)$ be a generalized metric space. Then we have $\mathcal{T}_{d} \subset \mathcal{T}^{d}$.

Proof. Let $U \in \mathcal{T}_{d}$. Suppose to the contrary that $U \notin \mathcal{T}^{d}$. Then there exists $x \in U$ such that $B\left(x, \frac{1}{n}\right) \not \subset U$ for all $n \in \mathbb{N}$. This implies that for each $n \in \mathbb{N}$, there exists $x_{n} \in B\left(x, \frac{1}{n}\right)$ and $x_{n} \notin U$. Since $d\left(x_{n}, x\right)<\frac{1}{n}$ for all $n \in \mathbb{N}, \lim _{n \rightarrow \infty} d\left(x_{n}, x\right)=0$. This proves that $\lim _{n \rightarrow \infty} x_{n}=x$ in $\left(X, \mathcal{T}_{d}\right)$. Therefore there exists $n_{0}$ such that $x_{n} \in U$ for all $n \geq n_{0}$. This is a contradiction of the fact that $x_{n} \notin U$ for all $n \in \mathbb{N}$.

The following example shows that inclusion in Proposition 2.1 can not be reversed.

Example 2.2. There exists a generalized metric space $(X, d)$ such that $\mathcal{T}^{d} \not \subset \mathcal{T}_{d}$

Proof. Let $(X, d)$ be the generalized metric space in Example 1.3. We have $\lim _{n \rightarrow \infty} d\left(\frac{1}{n}, 0\right)=\lim _{n \rightarrow \infty} \frac{1}{n}=0$. Then $\lim _{n \rightarrow \infty} \frac{1}{n}=0$ in $\left(X, \mathcal{T}_{d}\right)$. We also have $B\left(\frac{1}{3}, \frac{2}{3}\right)=\left\{x \in X: d\left(\frac{1}{3}, x\right)<\frac{2}{3}\right\}=\left\{0,2, \frac{1}{3}\right\}$. Then $B\left(\frac{1}{3}, \frac{2}{3}\right)$ is a neighborhood of 0 in $\left(X, \mathcal{T}^{d}\right)$. Since $\left\{\frac{1}{n}\right\}$ is not eventually in $B\left(\frac{1}{3}, \frac{2}{3}\right),\left\{\frac{1}{n}\right\}$ is not convergent to 0 in $\left(X, \mathcal{T}^{d}\right)$. Therefore, $\mathcal{T}^{d} \not \subset \mathcal{T}_{d}$. 
For the generalized metric spaces $\left(X, d_{X}\right)$ and $\left(Y, d_{Y}\right)$, we always consider $X \times Y$ to be the product space with respect to the mentioned topologies on $X$ and $Y$. Recall that, for a metric space $(X, d)$, the formula

$$
D\left(\left(x_{1}, y_{1}\right),\left(x_{2}, y_{2}\right)\right)=d\left(x_{1}, x_{2}\right)+d\left(y_{1}, y_{2}\right), x_{1}, x_{2}, y_{1}, y_{2} \in X
$$

yields a metric on $X \times X$. The following example shows that (in general), this is no longer true for a generalized metric space $(X, d)$.

Example 2.3. There exists a generalized metric space $(X, d)$ such that

$$
D\left(\left(x_{1}, y_{1}\right),\left(x_{2}, y_{2}\right)\right)=d\left(x_{1}, x_{2}\right)+d\left(y_{1}, y_{2}\right)
$$

for all $x_{1}, x_{2}, y_{1}, y_{2} \in X$, is not a generalized metric on $X \times X$.

Proof. Let $(X, d)$ be the generalized metric space in [9, Example 1.2]. We have

$$
\begin{aligned}
& D((1,1),(2,2)) \\
> & D\left((1,1),\left(1,1-\frac{1}{3}\right)\right)+D\left(\left(1,1-\frac{1}{3}\right),\left(2,1-\frac{1}{3}\right)\right)+D\left(\left(2,1-\frac{1}{3}\right),(2,2)\right) .
\end{aligned}
$$

Then $D$ is not a generalized metric on $X \times X$.

We state some relationships between a generalized metric and certain metric as follows.

Proposition 2.4. Let $(X, d)$ be a generalized metric space. If $\left(X, \mathcal{T}_{d}\right)$ has no isolated point and $d$ is a sequentially continuous function of its variables on $\left(X, \mathcal{T}_{d}\right) \times\left(X, \mathcal{T}_{d}\right)$, then $d$ is a metric on $X$.

Proof. For each $x \in X$, since $\left(X, \mathcal{T}_{d}\right)$ has no isolated point, we have $x \in$ $\overline{X-\{x\}}$ where $\overline{X-\{x\}}$ is the closure of $X-\{x\}$ in $\left(X, \mathcal{T}_{d}\right)$. For each sequence $\left\{x_{n}\right\} \subset X-\{x\}$ and $\lim _{n \rightarrow \infty} x_{n}=y$ in $\left(X, \mathcal{T}_{d}\right)$, if $y \neq x$, then $y \in X-\{x\}$. It implies that $X-\{x\}$ is sequentially closed in the sequential space $\left(X, \mathcal{T}_{d}\right)$. Then $X-\{x\}$ is closed in $\left(X, \mathcal{T}_{d}\right)$. Therefore, $\{x\}$ is open in $\left(X, \mathcal{T}_{d}\right)$, that is, $x$ is an isolated point of $\left(X, \mathcal{T}_{d}\right)$. It is a contradiction. Then there exists a sequence $\left\{x_{n}\right\} \subset X-\{x\}$ that $\lim _{n \rightarrow \infty} x_{n}=x$ in $\left(X, \mathcal{T}_{d}\right)$.

For each $x \neq y \neq z \in X$, choosing $z_{n} \in X-\{z\}$ for all $n \in \mathbb{N}$ such that $\lim _{n \rightarrow \infty} z_{n}=z$ in $\left(X, \mathcal{T}_{d}\right)$. We may assume that $z_{n} \in X-\{x, y, z\}$ for all $n \in \mathbb{N}$. Therefore,

$$
d(x, y) \leq d(x, z)+d\left(z, z_{n}\right)+d\left(z_{n}, y\right) .
$$

Taking the limit as $n \rightarrow \infty$ in (2.1) and using the assumption that $d$ is sequentially continuous in its variables on $\left(X, \mathcal{T}_{d}\right) \times\left(X, \mathcal{T}_{d}\right)$, we get $d(x, y) \leq$ $d(x, z)+d(z, y)$. This proves that $d$ is a metric on $X$. 
Proposition 2.5. Let $(X, d)$ be a generalized metric space. For each $x, y \in$ $X$, put

$$
\rho_{d}(x, y)= \begin{cases}0 & \text { if } x=y \\ \inf \left\{\max \left\{d\left(x, u_{1}\right), d\left(u_{1}, u_{2}\right), \ldots, d\left(u_{n}, y\right)\right\}:\right. & \\ \left.u_{1}, \ldots, u_{n} \in X, n \in \mathbb{N}\right\} & \text { if } x \neq y .\end{cases}
$$

Then we have

(1) $\rho_{d}$ is a metric on $X$.

(2) If $\lim _{n \rightarrow \infty} x_{n}=x$ in $(X, d)$, then $\lim _{n \rightarrow \infty} x_{n}=x$ in $\left(X, \rho_{d}\right)$.

Proof. 1. For all $x, y, z \in X$, we have $\rho_{d}(x, y) \geq 0, \rho_{d}(x, y)=\rho_{d}(y, x)$ and $\rho_{d}(x, y)=0$ if and only if $x=y$.

For each $\varepsilon>0$, there exist $u_{1}, \ldots, u_{n} \in X$ and $v_{1}, \ldots, v_{m} \in X$ such that

$$
\begin{aligned}
& \max \left\{d\left(x, u_{1}\right), d\left(u_{1}, u_{2}\right), \ldots, d\left(u_{n}, y\right)\right\}<\rho_{d}(x, y)+\frac{\varepsilon}{2} \\
& \max \left\{d\left(y, v_{1}\right), d\left(v_{1}, v_{2}\right), \ldots, d\left(v_{m}, z\right)\right\}<\rho_{d}(y, z)+\frac{\varepsilon}{2} .
\end{aligned}
$$

Then we have

$$
\begin{aligned}
\rho_{d}(x, z) \leq & \max \left\{d\left(x, u_{1}\right), d\left(u_{1}, u_{2}\right),\right. \\
& \left.\ldots, d\left(u_{n}, y\right), d\left(y, v_{1}\right), d\left(v_{1}, v_{2}\right), \ldots, d\left(v_{m}, z\right)\right\} \\
\leq & \max \left\{d\left(x, u_{1}\right), d\left(u_{1}, u_{2}\right),\right. \\
& \left.\quad \ldots, d\left(u_{n}, y\right)\right\}+\max \left\{d\left(y, v_{1}\right), d\left(v_{1}, v_{2}\right), \ldots, d\left(v_{m}, z\right)\right\} \\
\leq & \rho_{d}(x, y)+\frac{\varepsilon}{2}+\rho_{d}(y, z)+\frac{\varepsilon}{2} \\
= & \rho_{d}(x, y)+\rho_{d}(y, z)+\varepsilon .
\end{aligned}
$$

Therefore, $\rho_{d}(x, z) \leq \rho_{d}(x, y)+\rho_{d}(y, z)+\varepsilon$ for all $\varepsilon>0$. This proves that

$$
\rho_{d}(x, z) \leq \rho_{d}(x, y)+\rho_{d}(y, z) .
$$

By the above, $\rho_{d}$ is a metric on $X$.

2. Let $\lim _{n \rightarrow \infty} x_{n}=x$ in $(X, d)$. Then $\lim _{n \rightarrow \infty} d\left(x_{n}, x\right)=0$. If there exists $n_{0}$ such that $x_{n}=x$ for all $n \geq n_{0}$, then $\lim _{n \rightarrow \infty} x_{n}=x$ in $\left(X, \rho_{d}\right)$. So, we may assume that $x_{n} \neq x$ for all $n \in \mathbb{N}$. It implies that

$$
\begin{aligned}
0 & \leq \rho_{d}\left(x_{n}, x\right) \\
& =\inf \left\{\max \left\{d\left(x_{n}, u_{1}\right), d\left(u_{1}, u_{2}\right), \ldots, d\left(u_{m}, x\right)\right\}: u_{1}, \ldots, u_{m} \in X, m \in \mathbb{N}\right\} \\
& \leq d\left(x_{n}, x\right)
\end{aligned}
$$

for all $n \in \mathbb{N}$. Taking the limit as $n \rightarrow \infty$ in $(2.2)$, we get $\lim _{n \rightarrow \infty} \rho_{d}\left(x_{n}, x\right)=$ 0 . This proves that $\lim _{n \rightarrow \infty} x_{n}=x$ in $\left(X, \rho_{d}\right)$. 
Using [6, Proposition 2.2.4] and [6, Theorem 4.2.1], we get the following corollary from Proposition 2.4.

Corollary 2.6. Let $(X, d)$ be a generalized metric space. If $d$ is sequentially continuous in its variables on $\left(X, \mathcal{T}_{d}\right) \times\left(X, \mathcal{T}_{d}\right)$, then we have

(1) $\left(X, \mathcal{T}_{d}\right)=Y \oplus Z$, where $Y$ is metrizable and $Z$ is discrete. In particular, $\left(X, \mathcal{T}_{d}\right)$ is metrizable.

(2) $\left\{x_{n}\right\}$ is Cauchy in the generalized metric space $(X, d)$ if and only if $\left\{x_{n}\right\}$ is Cauchy in the metrizable space $\left(X, \mathcal{T}_{d}\right)$.

(3) The generalized metric space $(X, d)$ is complete if and only if the metrizable space $\left(X, \mathcal{T}_{d}\right)$ is complete.

Proof. (1) Denote $Y=\left\{x: x\right.$ is an isolated point of $\left.\left(X, \mathcal{T}_{d}\right)\right\}$ and

$$
Z=\left\{x: x \text { is not an isolated point of }\left(X, \mathcal{T}_{d}\right)\right\} .
$$

Then $X=Y \cup Z$ and $Y, Z$ are two disjoint open subsets of $\left(X, \mathcal{T}_{d}\right)$. By [6, Proposition 2.2.4], we have $X=Y \oplus Z$, where $Y$ is metrizable by Proposition 2.4 and $Z$ is discrete. Note that $Z$ is also a metrizable space with the discrete metric, then $\left(X, \mathcal{T}_{d}\right)$ is metrizable by [6, Theorem 4.2.1].

(2) We have that $\left\{x_{n}\right\}$ is Cauchy in the generalized metric space $(X, d)$ if and only if $\lim _{n, m \rightarrow \infty} d\left(x_{n}, x_{m}\right)=0$. It is equivalent to either $\left\{x_{n}\right\}$ is eventually in $Y$ or $\left\{x_{n}\right\}$ is eventually in $Z$, with respect to $\mathcal{T}_{d}$, and $\lim _{n, m \rightarrow \infty} d\left(x_{n}, x_{m}\right)=0$. Note that the metric on $Y$ is the restriction of the generalized metric $d$ on $Y$ and the metric on $Z$ is the discrete metric, then the above is equivalent to that $\left\{x_{n}\right\}$ is Cauchy in the metrizable space $\left(X, \mathcal{T}_{d}\right)$.

(3) It is a direct consequence of (1) and (2).

\section{Remark 2.7.}

(1) A discrete metric space is a counter-example showing that the converse of Proposition 2.4 is false.

(2) Let $(X, d)$ be an $n$-generalized metric space where $d$ is sequentially continuous in its variables on $\left(X, \mathcal{T}_{d}\right) \times\left(X, \mathcal{T}_{d}\right)$. As in the proofs of Proposition 2.4 and Corollary 2.6, we see that each $n$-generalized metric space $(X, d)$ reduces to either a discrete space or a metric space with the same Cauchy sequences and same completeness.

The following proposition presents the Hausdorff property of a generalized metric space.

Proposition 2.8. Let $(X, d)$ be a generalized metric space. Then we have

(1) If $d$ is sequentially continuous in its variables on $\left(X, \mathcal{T}_{d}\right) \times\left(X, \mathcal{T}_{d}\right)$, then $\left(X, \mathcal{T}_{d}\right)$ is Hausdorff.

(2) $\left(X, \mathcal{T}^{d}\right)$ is Hausdorff. 
Proof. (1) It is a direct consequence of Corollary 2.6.

(2) For each $x \neq y \in X$, we have $d(x, y)>0$. Put $r=\frac{d(x, y)}{2}$ and $U=B(x, r), V=B(y, r)$. Then $U$ is a neighborhood of $x$ and $V$ is a neighborhood of $y$ in $\left(X, \mathcal{T}^{d}\right)$ and $U \cap V=\emptyset$. This proves that $\left(X, \mathcal{T}^{d}\right)$ is a Hausdorff space.

The following example shows that the converse of Proposition 2.8.(1) is false.

Example 2.9. There exists a Hausdorff, generalized metric space $(X, d)$ such that $d$ is not sequentially continuous in its variables on $\left(X, \mathcal{T}_{d}\right) \times\left(X, \mathcal{T}_{d}\right)$.

Proof. Let $X=\{2\} \cup\left\{\frac{1}{n}: n \in \mathbb{N}\right\}$. Define $d: X \times X \longrightarrow \mathbb{R}$ as follows:

$$
d(x, y)= \begin{cases}0 & \text { if } x=y \\ 1 & \text { if } x=\frac{1}{n} \neq y=\frac{1}{m} ; n, m \in \mathbb{N} \\ =d(y, x)=y & \text { if } x=2, y=\frac{1}{n} ; n \in \mathbb{N} .\end{cases}
$$

Then $(X, d)$ is a generalized metric space. We have $\lim _{n \rightarrow \infty} d\left(\frac{1}{n}, 2\right)=$ $\lim _{n \rightarrow \infty} \frac{1}{n}=0$. Then $\lim _{n \rightarrow \infty} \frac{1}{n}=2$ in $\left(X, \mathcal{T}_{d}\right)$. Moreover, if $\lim _{m \rightarrow \infty} x_{m}=$ $\frac{1}{n}$ for some $n \in \mathbb{N}$ in $\left(X, \mathcal{T}_{d}\right)$, then $\lim _{m \rightarrow \infty} d\left(x_{m}, \frac{1}{n}\right)=0$. Then there exists $m_{0}$ such that $x_{m}=\frac{1}{n}$ for all $m \geq m_{0}$.

By the above, we have $U_{m}=\{2\} \cup\left\{\frac{1}{n}: n \geq m\right\}$ is a neighborhood of 2 in $\left(X, \mathcal{T}_{d}\right)$ for all $m \in \mathbb{N}$ and $V_{n}=\left\{\frac{1}{n}\right\}$ is a neighborhood of $\frac{1}{n}$ in $\left(X, \mathcal{T}_{d}\right)$ for all $n \in \mathbb{N}$. Since $U_{n+1} \cap V_{n}=\emptyset$ for all $n \in \mathbb{N}$ and $V_{n} \cap V_{m}=\emptyset$ for all $n \neq m$, we see that $\left(X, \mathcal{T}_{d}\right)$ is Hausdorff.

Since $\lim _{n \rightarrow \infty} \frac{1}{n}=2$ in $\left(X, \mathcal{T}_{d}\right)$ and $\lim _{n \rightarrow \infty} d\left(\frac{1}{2}, \frac{1}{n}\right)=\lim _{n \rightarrow \infty} 1=1 \neq$ $d\left(\frac{1}{2}, 2\right)=\frac{1}{2}$, we see that $d$ is not sequentially continuous in its variables on $\left(X, \mathcal{T}_{d}\right) \times\left(X, \mathcal{T}_{d}\right)$

An equivalent condition for a generalized metric $d$ to be sequentially continuous in its variables on $\left(X, \mathcal{T}_{d}\right) \times\left(X, \mathcal{T}_{d}\right)$ is as follows.

Proposition 2.10. Let $(X, d)$ be a generalized metric space. Then $d$ is sequentially continuous in its variables on $\left(X, \mathcal{T}_{d}\right) \times\left(X, \mathcal{T}_{d}\right)$ if and only if every convergent sequence on $\left(X, \mathcal{T}_{d}\right)$ is a Cauchy sequence on $(X, d)$.

Proof. Necessity. Let $\left\{x_{n}\right\}$ be a convergent sequence in $\left(X, \mathcal{T}_{d}\right)$. By using again notations in the proof of Corollary 2.6, we see that either $\left\{x_{n}\right\}$ is eventually in $Y$ or $\left\{x_{n}\right\}$ is eventually in $Z$, with respect to $\mathcal{T}_{d}$.

If $\left\{x_{n}\right\}$ is eventually in $Y$, then $\left\{x_{n}\right\}$ is a Cauchy sequence in $Y$ because $Y$ is a metric space. Note that the metric on $Y$ is the restriction of $d$ on $Y$, then $\left\{x_{n}\right\}$ is a Cauchy sequence in $(X, d)$.

If $\left\{x_{n}\right\}$ is eventually in $Z$, then there exists $n_{0}$ such that $x_{n}=x$ for all $n \geq n_{0}$ because $Z$ is discrete. Then $\left\{x_{n}\right\}$ is also a Cauchy sequence in $(X, d)$. 
By the above, $\left\{x_{n}\right\}$ is a Cauchy sequence in $(X, d)$.

Sufficiency. For each $x, y \in X$ and $\lim _{n \rightarrow \infty} x_{n}=x$ in $\left(X, \mathcal{T}_{d}\right)$, we will prove that $\lim _{n \rightarrow \infty} d\left(x_{n}, y\right)=d(x, y)$.

If $x=y$, then $\lim _{n \rightarrow \infty} d\left(x_{n}, y\right)=\lim _{n \rightarrow \infty} d\left(x_{n}, x\right)=0=d(x, x)=$ $d(x, y)$.

If there exists $n_{0}$ such that $x_{n}=x$ for all $n \geq n_{0}$, then $\lim _{n \rightarrow \infty} d\left(x_{n}, y\right)=$ $d(x, y)$.

So, we may assume that $x_{n} \neq x_{m} \neq x \neq y$ for all $n, m \in \mathbb{N}$. Then

$$
\begin{aligned}
d\left(x_{n}, y\right) & \leq d\left(x_{n}, x_{m}\right)+d\left(x_{m}, x\right)+d(x, y), d(x, y) \\
& \leq d\left(x, x_{m}\right)+d\left(x_{m}, x_{n}\right)+d\left(x_{n}, y\right) .
\end{aligned}
$$

It implies that

$$
\begin{aligned}
d\left(x_{n}, y\right)-d(x, y) & \leq d\left(x_{n}, x_{m}\right)+d\left(x_{m}, x\right), d(x, y)-d\left(x_{n}, y\right) \\
& \leq d\left(x, x_{m}\right)+d\left(x_{m}, x_{n}\right) .
\end{aligned}
$$

Therefore, we have

$$
\left|d\left(x_{n}, y\right)-d(x, y)\right| \leq d\left(x_{n}, x_{m}\right)+d\left(x_{m}, x\right)
$$

for all $n \in \mathbb{N}$. Taking the limit as $n \rightarrow \infty$ in (2.3), note that $\left\{x_{n}\right\}$ is a Cauchy sequence, we get $\lim _{n \rightarrow \infty}\left|d\left(x_{n}, y\right)-d(x, y)\right|=0$. That is, $\lim _{n \rightarrow \infty} d\left(x_{n}, y\right)=$ $d(x, y)$.

The proof of Sufficiency of Proposition 2.10 gives the following result.

Corollary 2.11 ([11], Proposition 3). Let $(X, d)$ be a generalized metric space. If $\left\{x_{n}\right\}$ is a Cauchy sequence and $\lim _{n \rightarrow \infty} x_{n}=x$ in $\left(X, \mathcal{T}_{d}\right)$, then $\lim _{n \rightarrow \infty} d\left(x_{n}, y\right)=d(x, y)$ for all $y \in X$. In particular, $\lim _{n \rightarrow \infty} x_{n} \neq y$ in $\left(X, \mathcal{T}_{d}\right)$ if $y \neq x$.

Remark 2.12. Recently, the authors of [9] asserted that there were some incorrect proofs in [1], [2], [3], [12] by using the 'false' Proposition 1.2. These 'false properties' of generalized metric spaces were first observed by Das and Dey in [4], [5]. Also, these facts were observed by Samet in [13], by Lakzian and Samet in [14], by Sarma et al. in [15]. A fact first noted in [16], and then in [11] that the Hausdorff property in [15, Theorem 1.3] is superfluous. By using Corollary 2.11, we also see that the Hausdorff property in [14, Theorem 3.1], [14, Theorem 3.2], [7, Theorem 4], [7, Theorem 9], [7, Theorem 11], [7, Theorem 13] are superfluous. Therefore, the comments in [9] on the proofs in [1], [2], [3], [12] may be not fair, in the sense that Corollary 2.11 is used implicitly.

Now we restate Example 1.3 as follows, also for [9, Example 1.2].

Example 2.13. Let $(X, d)$ be the complete generalized metric space in Example 1.3. Then we have 
(1) $\left(X, \mathcal{T}^{d}\right)$ is discrete. In particular, $\left(X, \mathcal{T}^{d}\right)$ is Hausdorff and $\left\{\frac{1}{n}\right\}$ is not convergent in $\left(X, \mathcal{T}^{d}\right)$.

(2) $\lim _{n \rightarrow \infty} \frac{1}{n}=0$ and $\lim _{n \rightarrow \infty} \frac{1}{n}=2$ in $\left(X, \mathcal{T}_{d}\right)$. In particular, $\left(X, \mathcal{T}_{d}\right)$ is not Hausdorff.

(3) The sequence $\left\{\frac{1}{n}\right\}$ is not a Cauchy sequence in $(X, d)$.

(4) The collection $\mathcal{B}=\{B(x, r): r>0\}$ does not form a neighborhood basis at $x$ in $\left(X, \mathcal{T}^{d}\right)$.

(5) $d$ is not a sequentially continuous function of its variables on $\left(X, \mathcal{T}_{d}\right) \times$ $\left(X, \mathcal{T}_{d}\right)$.

(6) $d$ is continuous on $\left(X, \mathcal{T}^{d}\right) \times\left(X, \mathcal{T}^{d}\right)$.

Proof. For (3), see Example 1.3.(3); (4) is a direct consequence of Example 1.3.(5) and (6) is a direct consequence of (1).

(1) We have $B\left(0, \frac{1}{3}\right) \cap B\left(\frac{1}{3}, \frac{2}{3}\right)=\{0\}, B\left(2, \frac{1}{3}\right) \cap B\left(\frac{1}{3}, \frac{2}{3}\right)=\{2\}, B\left(\frac{1}{n}, r\right)=$ $\left\{\frac{1}{n}\right\}$ if $r<\frac{1}{n}$. It implies that $\left(X, \mathcal{T}^{d}\right)$ is discrete.

(2) We see that $\lim _{n \rightarrow \infty} d\left(\frac{1}{n}, 0\right)=\lim _{n \rightarrow \infty} d\left(\frac{1}{n}, 2\right)=\lim _{n \rightarrow \infty} \frac{1}{n}=0$. Then $\lim _{n \rightarrow \infty} \frac{1}{n}=0$ and $\lim _{n \rightarrow \infty} \frac{1}{n}=2$ in $\left(X, \mathcal{T}_{d}\right)$. Therefore, $\left(X, \mathcal{T}_{d}\right)$ is not Hausdorff by [6, Proposition 1.6.7].

(5) We have $\lim _{n \rightarrow \infty} \frac{1}{n}=0$ in $\left(X, \mathcal{T}_{d}\right)$ but $\lim _{n \rightarrow \infty} d\left(\frac{1}{n}, 2\right)=\lim _{n \rightarrow \infty} \frac{1}{n}=$ $0 \neq d(0,2)=1$. Then $d$ is not a sequentially continuous function of its variables on $\left(X, \mathcal{T}_{d}\right) \times\left(X, \mathcal{T}_{d}\right)$.

In [5], Das and Dey introduced the notion of a generalized normed linear space as follows.

Definition 2.14 ([5]). Let $X$ be a real or complex vector space over the field $\mathbb{K}$ and $\|\cdot\|: X \longrightarrow \mathbb{R}$ such that

(1) $\|x\| \geq 0$ and $\|x\|=0$ if and only if $x=0$ for all $x \in X$.

(2) $\|\lambda . x\|=|\lambda| .\|x\|$ for all $x \in X$ and $\lambda \in \mathbb{K}$.

(3) $\left\|x+z_{1}+\ldots+z_{k}+y\right\| \leq\|x\|+\left\|z_{1}\right\|+\ldots+\left\|z_{k}\right\|+\|y\|$ for all $x, y, z_{1}, \ldots, z_{k} \neq 0$.

Then the function $\|$.$\| is called a generalized norm on X$ and $(X,\|\|$.$) is called$ a generalized normed linear space. If $(X,\|\|$.$) is a generalized normed linear$ space, then $d(x, y)=\|x-y\|$ for all $x, y \in X$ is a generalized metric on $X$ and $d$ is called the generalized norm induced by $\|$.$\| . A generalized normed$ linear space which is complete with respect to the induced generalized metric is called a generalized Banach space.

The following proposition shows that every generalized norm is a norm. Then, all results and open problems in [5] are redundant.

Proposition 2.15. If $(X,\|\cdot\|)$ is a generalized normed linear space, then the function $\|\cdot\|$ is a norm on $X$, that is, $\|x+y\| \leq\|x\|+\|y\|$ for all $x, y \in X$. 
Proof. If $x=0$ or $y=0$, then we have $\|x+y\| \leq\|x\|+\|y\|$. So we may assume that $x, y \neq 0$. Then we have

$$
\begin{aligned}
\|x+y\| & =\left\|x+\frac{1}{2} y+\frac{1}{2} y\right\| \\
& \leq\|x\|+\left\|\frac{1}{2} y\right\|+\left\|\frac{1}{2} y\right\|=\|x\|+\frac{1}{2}\|y\|+\frac{1}{2}\|y\|=\|x\|+\|y\| .
\end{aligned}
$$

By the above, $\|\cdot\|$ is a norm on $X$.

Acknowledgments. The first author would like to thank the King Mongkut's University of Technology Thonburi (KMUTT) for financial support. The second author would like to thank the Dong Thap Seminar on Mathematical Analysis and its Applications for discussion about the paper.

\section{REFERENCES}

[1] A. Branciari, A fixed point theorem of Banach-Caccioppoli type on a class of generalized metric spaces, Publ. Math. Debrecen, 57 (1-2) (2000), 31-37.

[2] P. Das, A fixed point theorem on a class of generalized metric spaces, Korean J. Math. Sci., 1 (2002), 29-33.

[3] P. Das and L. K. Dey, A fixed point theorem in a generalized metric space, Soochow J. Math., 33 (2007), 33-29.

[4] P. Das and L. K. Dey, Fixed point of contractive mappings in generalized metric spaces, Math. Slovaca, 59 (4) (2009), 499-504.

[5] P. Das and L. K. Dey, Porosity of certain classes of operators in generalized metric spaces, Demonstratio Math., XLII (1) (2009), 163-174.

[6] R. Engelking, General Topology, Sigma series in pure mathematics, vol. 6, Heldermann Verlag, Berlin, 1988.

[7] I. M. Erhan, E. Karapinar, and T. Sekulic, Fixed points of $(\psi, \phi)$ contractions on generalized metric spaces, Fixed Point Theory Appl., 2012:138 (2012), 1-10.

[8] S. P. Franklin, Spaces in which sequences suffice, Fund. Math., 57 (1965), 107-115.

[9] L. Kikina and K. Kikina, On fixed point of a Ljubomir Ciric quasi-contraction mapping in generalized metric spaces, Publ. Math. Debrecen, 83 (3) (2013), 1-6.

[10] L. Kikina and K. Kikina, A fixed point theorem in generalized metric spaces, Demonstratio Math., XLVI (1) (2013), 181-190.

[11] W. A. Kirk and N. Shahzad, Generalized metrics and Caristi's theorem, Fixed Point Theory Appl., 2013:129 (2013), 1-9.

[12] B. K. Lahiri and P. Das, Fixed point of a Ljubomir Ciric's quasi-contraction mapping in a generalized metric space, Publ. Math. Debrecen, 61 (3-4) (2002), 589-594.

[13] B. Samet, Discussion on "A fixed point theorem of Banach-Caccioppoli type on a class of generalized metric spaces" by A. Branciari, Publ. Math. Debrecen, 76 (4) (2010), 493-494.

[14] H. Lakzian B. Samet, Fixed points for $(\psi, \varphi)$-weakly contractive mappings in generalized metric spaces, Appl. Math. Lett., 25 (2012), 902-906.

[15] I. R. Sarma, J. M. Rao, and S. S. Rao, Contractions over generalized metric spaces, J. Nonlinear Sci. Appl., 2 (3) (2009), 180-182. 
[16] M. Turinici, Functional contractions in local Branciari metric spaces, ROMAI J., 8 (2) (2012), 189-199.

(Received: November 25, 2013)

(Revised: January 27, 2014)
Poom Kumam

Department of Mathematics

Faculty of Science

King Mongkut's University of

Technology Thonburi (KMUTT)

Bang Mod, Thrung Khru

Bangkok 10140

Thailand

poom.kum@kmutt.ac.th

Nguyen Van Dung

Faculty of Mathematics and Information

Technology Teacher Education

Dong Thap University

Cao Lanh City, Dong Thap Province

Viet Nam

nvdung@dthu.edu.vn 